\title{
Probing student understanding of spectra through the use of a typical experiment used in teaching introductory modern physics
}

\author{
Lana Ivanjek $\odot,{ }^{1, *}$ Peter Shaffer $\odot,{ }^{2}$ Maja Planinić $\odot,{ }^{3}$ and Lillian $\mathrm{McDermott}^{2}$ \\ ${ }^{1}$ University of Vienna, Faculty of Physics, Porzellangasse 4, 1090 Vienna, Austria \\ ${ }^{2}$ University of Washington, Department of Physics, Seattle, 98195-1560 Washington, USA \\ ${ }^{3}$ Department of Physics, Faculty of Science, University of Zagreb, Bijenicka c. 32, 10000 Zagreb, Croatia
}

(Received 21 August 2019; published 8 January 2020)

\begin{abstract}
The topic of atomic spectra is part of university and secondary school curricula around the world. Relatively little research, however, has been done on the learning and teaching of this subject, despite the fact that it forms a foundation for advanced study in quantum mechanics, astronomy, and astrophysics. A systematic investigation into student understanding of the formation and structure of atomic spectra was conducted among more than 1000 science majors in physics courses at the University of Zagreb, Croatia and the University of Washington, USA. The research had two primary goals: (i) to probe the extent to which university students are able to relate the wavelength of spectral lines to the transition of electrons between energy levels in an atom, and (ii) to probe the extent to which students recognize the conditions under which discrete line spectra are (and are not) formed. This paper focuses on the latter aspect, in particular, student understanding of the experimental setup that is commonly used to illustrate the formation of discrete line spectra. Students were asked about how changes to a setup consisting of a light source, a mask with a slit, a prism (or diffraction grating), and a screen affect the spectra observed. The findings suggest that relatively few students recognize that the type of light source is critical for the formation of line spectra. Instead students often attribute the formation of line spectra to the slit, the prism (or diffraction grating), or even to the distance between the prism and screen.
\end{abstract}

DOI: 10.1103/PhysRevPhysEducRes.16.010102

\section{INTRODUCTION}

This paper presents results from an investigation into student understanding of atomic emission spectra conducted in collaboration by the physics education groups at the University of Zagreb, Zagreb, Croatia and the University of Washington (UW), Seattle, Washington, USA. A major purpose has been to probe the extent to which university students understand the mechanism underlying the formation and structure of discrete spectral lines. In two previous papers, we demonstrated difficulties students have in relating spectral lines to transitions of electrons between energy levels. [1,2] This paper focuses on another aspect-student understanding of the role of each component of the experimental setup in forming atomic spectra lines.

Historically, the observation of discrete spectra was a key part of the motivation for the development of quantum

\footnotetext{
*lana.ivanjek@univie.ac.at

Published by the American Physical Society under the terms of the Creative Commons Attribution 4.0 International license. Further distribution of this work must maintain attribution to the author(s) and the published article's title, journal citation, and DOI.
}

mechanics. Observations of the spectra (continuous and discrete) that result from various experimental setups and light sources lay the groundwork for understanding the important ideas of electron levels and quantization of energy.

In a typical course, observations of discrete line spectra and the idea of atomic energy levels are introduced after students have completed the study of geometrical and physical optics. Students have previously observed the interference patterns that arise when light of a single wavelength (e.g., from a laser) passes through one, two, or more slits, including diffraction gratings. They have also observed the continuous spectrum that results when a thin beam of white light passes through a prism and is incident on a screen. (Sometimes a diffraction grating is used instead.) This observation motives the idea that white light consists of many wavelengths. At this point, the light source is changed to a gas discharge tube (e.g., a fluorescent lamp) and students observe only a few discrete colored lines on the screen. The focus then shifts to the atoms in the gas and a model is developed in which transitions of electrons between discrete energy levels account for the formation of only certain, discrete, wavelengths of light.

We have found, however, that students often fail to interpret observations of discrete line spectra in terms of 
the model discussed above. Even after all instruction (including lecture demonstrations and laboratory experiments), many fail to associate the formation of discrete spectra with the type of light source. Some students seem to believe that such spectra result from diffraction. Many do not understand the role of the various devices in the experimental setup (the slit, prism, and diffraction grating). Even how the pattern changes when the distance between the mask and the screen is varied may not be understood.

The research presented in this paper was motivated by informal observations of junior physics majors in laboratory courses at the University of Zagreb and in interviews conducted with nine of these students. Although the interviews focused on student ability to associate the line spectra with electron transitions [1], some of the responses suggested a failure of students to recognize that a discrete spectrum can only result from certain light sources. Many predicted that a discrete spectrum could be formed from an incandescent bulb if certain changes were made to the experimental apparatus (e.g., if the prism were replaced by a diffraction grating).

The results presented in this paper are drawn from classes in which lectures on atomic spectra had been completed and students had observed spectra in lecture demonstrations. Some of the students had also completed laboratory experiments in which they had observed line spectra. (See Fig. 1 for a common demonstration or experiment used to observe atomic line spectra.)

\section{PRIOR RESEARCH}

There are relatively few studies on student understanding of spectroscopy. One that is relevant to this paper was conducted at Kansas State University. The study probed the ability of 67 elementary education majors to observe and describe spectra. The findings indicated that students who did not have prior experience in observing spectra tended to interpret the hydrogen spectrum as being continuous, rather than discrete [3].

Other studies have probed student reasoning about physical and geometrical optics. The UW Physics Education Group had previously examined university student

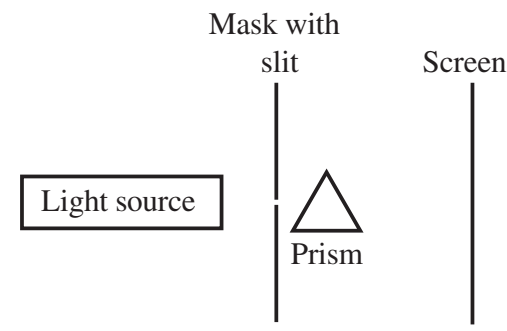

FIG. 1. Common experiment used to view discrete line spectra. If the source is an incandescent bulb, a continuous spectrum is observed. A gas discharge lamp yields a discrete line spectrum. In some cases, a diffraction grating is used instead of a prism. understanding of physical optics and found that many students at introductory and more advanced levels do not develop a coherent model that they can use to predict and explain interference and diffraction effects [4-7]. Ward et al. [8] investigated children's understanding of light and found that certain aspects of the dispersion of light through a prism present difficulties, especially for younger children. One common incorrect idea they found is that children often believe that "white" light has no color and that a prism adds color to light [8]. Anderson examined how 5th grade students reasoned about the nature of white light and found that the vast majority (72 of 100) regarded white light as being clear or colorless. Many of these students also thought that color is a property of an object, rather than being associated with light [9].

In addition to specific research on light and spectroscopy, there is a growing body of research on demonstrations and labs showing that these are often ineffective in promoting student learning of the intended physical concepts-and may even be counterproductive [10-13]. Crouch et al. [14], for example, found no significant difference between students who had and had not observed certain demonstrations. Kraus [15] found that after traditional lecture demonstrations students often do not correctly recall the results or make incorrect observations during the demonstration. Miller et al. [16] found that roughly one out of every five observations of a demonstration is inconsistent with the actual outcome and that students who understand the underlying concepts before observing the demonstration or who are asked to predict the outcome first are more likely to observe what is needed and remember it correctly. This is consistent with the work of Thornton and Sokoloff [17] and Kraus [15] who showed that replacing traditional lectures with interactive lecture demonstrations or tutorial lectures can improve conceptual understanding.

Even laboratory experiments that, on the surface, appear designed to engage students in their learning may not have an impact on student conceptions. Wiemann and Holmes [10] and Holmes et al. [11] analyzed the effect of taking a lab course on student learning in introductory calculusbased physics courses. The labs were well coordinated with the lectures and included prelab activities that consisted of sequences of questions that led students to make predictions and explore the relevant physics concepts. They found no significant difference in final exam performance by students who had or had not completed the laboratory $[10,11]$. They note, however, that this result does not rule out the possibility that there are other things (e.g., lab skills) that are learned in the lab but that are not tested for by course examinations.

An investigation involving senior-level students in an Australian high school physics course [18] attempted to identify some of the reasons why students do not learn from traditional demonstrations what instructors intend. They identified six issues that may prevent students from 
learning in traditional lecture settings. These include (i) lack of a theoretical framework that can help students separate the phenomena from other factors (noise), (ii) "interference of discourses" taught in other contexts of the course, (iii) interference from other demonstrations and images that have surface resemblance, (iv) problems in piecing together coherent representational frameworks from the information given, (v) low salience of knowledge related to demonstrations on tests, and (vi) lack of opportunities for students to test their descriptions and explanations. In addition, Northedge [19] claims that university teachers' thoughts are often so deeply rooted in the discourse of specialists that they are unaware that meanings they take for granted are simply not construable from outside the discourse. Along these lines Fredlund et al. [20] observed students engaged in a laboratory exercise on $R C$ circuits and examined how they used a circuit diagram to construct a circuit and then interpreted their observations of the circuit behavior. The authors speculate that "rationalized" representations, as, for example, circuit diagrams, are powerful communicative resources for physicists, but can constitute significant learning and teaching challenges to students because students are not aware of the critical underlying physics aspects of a particular phenomenon, situation, or construct. As proposed by Fredlund et al. [20], instructors need to help students unpack the representations, guide them in making the appropriate connections to the physics concepts, and check that the representations effectively promote conceptual understanding.

This prior research is consistent with a view that student thinking in physics can be regarded as arising from a set of loosely connected knowledge and reasoning elements that may be applied in a highly context-dependent manner [21-23]. A response to a given question may not reflect a coherent conceptual framework, but rather be based on prior knowledge or experience that may or may not be directly relevant. Student responses can also draw on basic reasoning elements that are, in and of themselves, neither correct nor incorrect (e.g., phenomenological primitives or $p$ prims) [21]. Application of these different cognitive resources may depend on how students, consciously or unconsciously, interpret (or frame) the question. Responses may even be based on perceptions of the cultural expectations surrounding the administration of the question [23]. These considerations are likely especially relevant for topics that are abstract or divorced from everyday experience, such as is the case for spectroscopy. In conducting this research, we therefore tried to probe explicitly how students interpret what they see on the screen and how they relate their observations to the experimental setup.

\section{DATA COLLECTION AND ANALYSIS}

The results presented in this paper are based on student responses to two written tasks that were constructed to probe student thinking about the conditions required to obtain a discrete spectrum. Both tasks involve an experiment in which changes are made to an experimental setup consisting of a light source, a mask with a slit, a prism, and a screen.

The development of the tasks was motivated, in part, by informal observations of students as they worked through a traditional laboratory experiment on continuous and discrete spectroscopy as well as by results from 9 semistructured demonstration interviews conducted with junior physics majors at the University of Zagreb. The interviews, which were conducted after all relevant instruction, primarily focused on student ability to associate line spectra with electron transitions (the subject of two earlier papers $[1,2]$.) However, each interview began by showing students an experimental setup similar to what they had used in the lab and the continuous spectrum that would appear on the screen. They were asked if, and how, they could obtain a discrete spectrum instead of a continuous one. Only 4 of the students correctly stated that a discrete spectrum arises from certain types of light sources. The others described changes they could make to other parts of the apparatus. We interpreted the responses, together with the observations we had made during the labs, to suggest that many students had failed to reflect upon and to understand the role of each piece of equipment in the experiment. Tasks 1 and 2 were the result of our attempt to probe the underlying student conceptions and their prevalence.

Task 1: In the first task, students are told that the light source is an incandescent bulb and are shown the continuous spectrum that would result on the screen (see Fig. 2). They are then given a list of possible changes to the equipment and asked to identify which change (or changes) could result in a discrete, rather than continuous spectrum and to explain their reasoning. (In some cases, students were also given a diagram showing a discrete line spectrum to ensure that they knew what was meant by that term. The inclusion of the discrete spectrum had no impact on the results.) The correct answer is that a discrete spectrum can only be obtained if the incandescent bulb is replaced by a different light source (e.g., a mercury lamp that emits light of discrete wavelengths).

Task 2: In the second task, students are told that light from a gas-discharge light source (e.g., a mercury lamp) passes through a slit and a glass prism before it is incident on a screen. The resulting discrete line spectrum is shown (see Fig. 3). Students are told that the prism is then removed and asked how the spectrum on the screen would change and to explain their reasoning. (They answered by selecting the correct answer from a list of proposed changes.) Students were expected to reason that, without the prism, all the light that passes through the slit would follow a straight-line path directly to the screen. Thus, there would only be a single bright spot at the center. (The color that they predicted for the bright spot was not considered in judging whether or not a student response was correct.) 

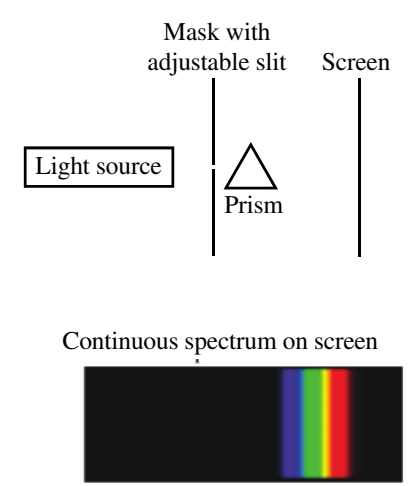

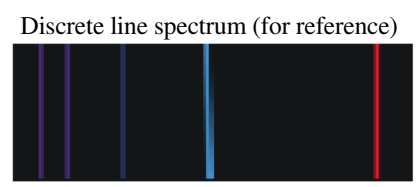

FIG. 2. Task 1. Question administered to all populations of students. In some cases, the question did not ask for explanations of reasoning.

Data for the present study were obtained from physics courses at three universities: the University of Washington, Seattle (UW), the University of Zagreb, Croatia (UZ), and the University of Vienna, Austria (UV). In all cases, students had completed the relevant lecture and laboratory instruction.

The undergraduate students at UW were enrolled in either the standard introductory calculus-based course (UW Intro, $N=1330$ ) or an "honors" section of the same course (UW Honors, $N=176$ ). (The honors section involves a select group of students, not necessarily physics majors,

The picture below shows a portion of the discrete line spectrum that appears on a screen. The spectrum is obtained by using a setup consisting of a gas discharge light source, a single slit, and a glass prism.

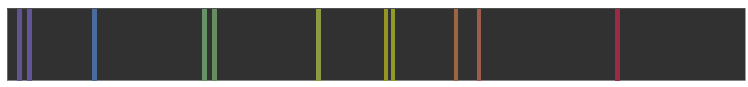

What would happen if the prism were removed? Explain.

A. The lines would become more closely spaced.

B. The lines would remain the same.

C. The lines would stay at the same location, but all would have the same color.

D. The lines would vanish and be replaced by a single bright spot at the center of the screen.

FIG. 3. Task 2. The UW version included another choice, E, which stated that a bright region would appear where the lines had been located. Student explanations were essentially the same as for choice D, therefore choice $\mathrm{E}$ was not used at the other institutions. Some version of the question did require explanations of reasoning. who are interested in pursuing a rigorous interdisciplinary program of study.) Both groups had completed two prior quarters courses on mechanics and electromagnetism, and were enrolled in a third course on waves, optics, and modern physics. Instruction was primarily through lecture ( $3 \mathrm{~h} /$ week), lab ( $2 \mathrm{~h} /$ week), and small group tutorials (1 h/week) [24]. Some of the UW data also come from graduate and undergraduate teaching assistants in a teaching seminar.

At the University of Zagreb, the populations included second-year physics majors in an introductory calculusbased physics course $(N=116)$ and junior-level physics majors $(N=36)$. The second-year students had completed calculus, General Physics 1-3 (mechanics, electromagnetism, and waves and optics), and were enrolled in General Physics 4, which covers thermal and modern physics. They had also completed labs that included experiments on geometrical and physical optics. The junior-level students had completed a course on quantum mechanics.

The students at the University of Vienna $(N=74)$ were similar to the second-year students at the University of Zagreb. They had previously completed General Physics, which included mechanics, electromagnetism, and optics, and were enrolled in a course that covered modern physics. Most had also completed introductory labs that included optics and spectroscopy.

Both tasks 1 and 2 were administered after all lectures on spectra had been completed but before students had worked through a tutorial designed to help them relate discrete spectral lines to transitions between energy levels [2]. The tasks were given on paper or as part of an online survey. In most cases, students were asked to give explanations. All students at the University of Washington wrote (or typed) their explanations. No explanations were asked of 
students at the University of Vienna and only some of the students from the University of Zagreb were asked to give explanations.

All the explanations were carefully read and categorized based on general guidelines for analyzing verbalized data [25]. First, all the explanations were read to try to get insight into student thinking and to identify the most frequent strategies used to answer the tasks. Based on that, a preliminary set of categories was proposed. The data were then coded based on these categories. The categories were refined and new ones added as needed. Sometimes a student response suggested more than one strategy-in which case, it was assigned to each. The most common strategies are discussed in the next section.

\section{RESULTS AND DISCUSSION}

Typically, the different sections of each course at a given university were taught by different instructors, who used different demonstrations and had different approaches for engaging students during lecture. Yet, across the different sections, the range in the percentage of students answering correctly was typically no greater than $10 \%$ to $15 \%$. It is possible that the few larger differences might be attributed to the different instructors, the use of specific demonstrations, or to the fact that, in some cases, students had received somewhat different instruction on physical optics. (For example, some of the classes had worked through the physical optics section of Tutorials in Introductory Physics [24].) However, a comparison of the percentages across the classes is not the focus of this paper. Rather, the emphasis is on the analysis of strategies that students used to illustrate the formation of discrete line spectra, when asked about the role of the devices in the experimental setups.

\section{A. Student performance}

Summaries of the results from tasks 1 and 2 are given in Tables I and II. Across the three universities, at most $40 \%$ of the introductory students recognized after lecture instruction that the only way to produce a discrete spectrum, instead of a continuous one, is to change the light source (see Table I). Similarly, only about two-thirds of the students recognized how the discrete line spectrum in task 2 would change if the prism were removed. (See Table II.) The implication is that after instruction, many students fail to understand the features that characterize a discrete line spectrum and do not recognize the unique role of the light source in generating a discrete spectrum.

\section{B. Discussion of student strategies}

The tasks used in this study were primarily designed to assess whether students recognize that only a change in the light source can lead to discrete atomic spectra. The tasks, however, also probe student ability to relate the changes in the experimental setup to the changes in the pattern that

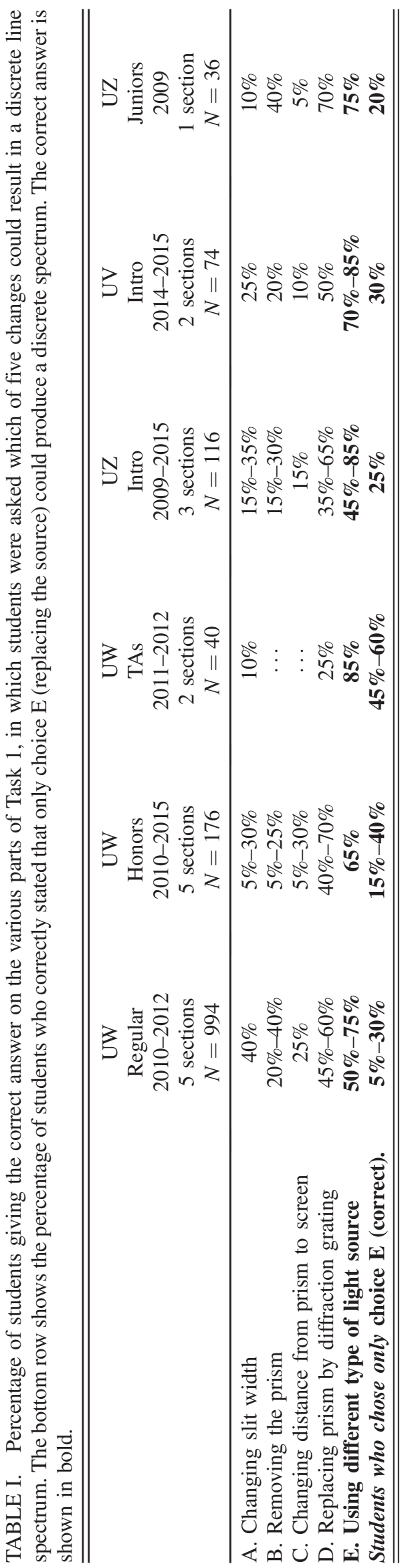


TABLE II. Results from Task 2, in which students were asked how the discrete spectral line pattern would change if the prism were removed from the optical set-up. The correct answer is shown in bold.

\begin{tabular}{|c|c|c|c|c|}
\hline & $\begin{array}{c}\text { UW } \\
\text { Regular } \\
\text { 2009-2010 } \\
2 \text { sections } \\
N=336\end{array}$ & $\begin{array}{c}\text { UZ } \\
\text { Intro } \\
2009,2015 \\
2 \text { sections } \\
N=50\end{array}$ & $\begin{array}{c}\text { UZ } \\
\text { Juniors } \\
2009 \\
1 \text { section } \\
N=36\end{array}$ & $\begin{array}{c}\text { UV } \\
\text { Intro } \\
2014 \\
1 \text { section } \\
N=44\end{array}$ \\
\hline A. The lines would become more closely spaced & $10 \%-20 \%$ & $35 \%$ & $5 \%$ & $15 \%$ \\
\hline $\begin{array}{l}\text { B/C. The lines would remain same or would } \\
\text { have the same color }\end{array}$ & $5 \%-15 \%$ & $0 \%-10 \%$ & $15 \%$ & $5 \%$ \\
\hline $\begin{array}{l}\text { D. The lines would be replaced by single } \\
\text { bright spot at center }\end{array}$ & $60 \%-75 \%$ & $45 \%-65 \%$ & $70 \%$ & $75 \%$ \\
\hline Other $^{*}$ (diffraction pattern) & $10 \%$ & $\ldots$ & $\ldots$ & $\ldots$ \\
\hline
\end{tabular}

"In the UW version, students had the option of selecting "other" and giving a description. About 10\% of the students stated a diffraction pattern would appear on the screen.

would be observed on the screen. To answer correctly, students need to be able to apply a range of concepts that span geometrical and physical optics and modern physics. They must recognize the role of the individual optical elements (the light source, the mask, and the prism or diffraction grating) in generating the pattern. Important ideas include recognizing that (i) white light consists of a continuous range of wavelengths, (ii) the type of source determines the type of spectrum, (iii) the slit serves to form a narrow beam (ray) of light, (iv) a prism serves to separate the various frequencies of light present in a narrow beam, and (v) a diffraction grating creates a pattern through the interference of light of each frequency. We found that students had difficulty with each of these steps within the context of this experiment. Their explanations provided insight not only into their ideas about light but also into how they thought about the role and function of each optical device.

All of the incorrect reasoning patterns that we identified are summarized in Table III. These range from confusion between a discrete line spectrum and a diffraction pattern to treating a prism as "adding color" to light. The table shows the extent to which each reasoning pattern was elicited by each change to the devices in the experimental setup. Some of the reasoning patterns are tied to a specific device, while others are associated with multiple devices.

Possibly the most significant finding is that many students do not associate the formation of discrete line spectra only with the light source. Many seem to believe that it is possible to obtain a discrete spectrum from a continuous one by changing elements of the experimental setup besides the light source. The last line in Table I shows that $80 \%$ or more of the introductory students gave responses in which they treated the prism, the slit, the diffraction grating, or the prism-screen distance as potentially yielding a discrete line spectrum. Some of the responses reflect a failure of students to recognize the specific role of each element in the experiment, others reveal how students think about light more generally (for example, a tendency of students to treat a continuous spectrum as consisting of a finite set of colors).

The most common incorrect student strategies are discussed below. They include responses from both of the populations that were asked for explanations (UW and UZ). The strategies are organized according to the optical instrument or the aspect of the experimental setup that elicited them (slit, prism, grating, mask-screen distance, and light source). When a specific pattern of reasoning was associated with more than one of the devices, it is discussed only in the section about the device that elicited that strategy most strongly. Exceptions are made when there are aspects of the reasoning brought out by other devices. Note that the strategies are not mutually exclusive; in some cases, individual student responses can be interpreted in multiple ways.

\section{Reasoning elicited by changing the slit width (question 1A)}

Question 1A probes student understanding of the role of the slit. Students are asked whether altering the slit width could change the observed continuous spectrum into a discrete line spectrum. Only about $60 \%$ of the students answered correctly, with $60 \%$ of them giving correct reasoning. Some of them correctly stated that only changing the light source could change the type of spectrum.

"No [a discrete line spectrum would not occur] because light source of incandescent light bulb still produces continuous spectrum no matter what factor we change unless the light source is replaced by light source that can produce discrete spectrum such as hydrogen lamp." [UW Intro, task 1]

This statement alone would be enough to answer every part of task 1 . Other students who answered correctly went into detail about how changing the slit width would widen or narrow the spectrum (in the geometrical optic limit), or how it would generate a complicated pattern of continuous 


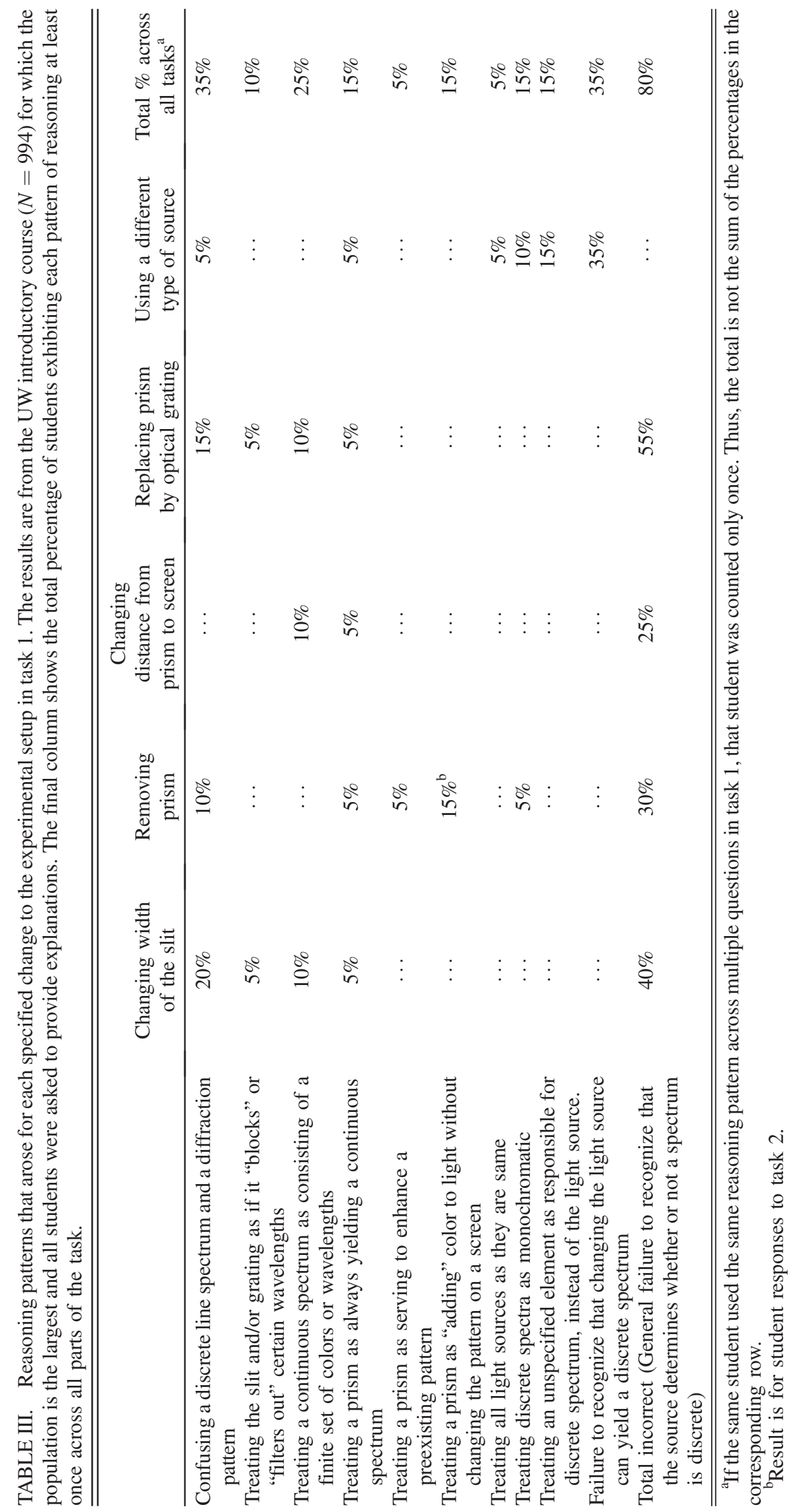


spectra with interference (if diffraction occurs). Both of these types of responses were treated as correct.

On question 1A, about $40 \%$ of the students failed to recognize that the primary role of the slit in this experiment is to columnate the light into a narrow beam. Their explanations indicated various incorrect ideas. For example, (a) some students seemed to think that the slit was producing a diffraction pattern and confused that pattern with a discrete line spectrum. (b) Others predicted that a discrete line spectrum would result since a narrower slit would "block" certain wavelengths. (c) Often students seemed to treat the continuous spectrum that was shown to them as if it were already discrete and predicted that its discrete nature would become more apparent if the slit size were changed. Question 1A about the slit also elicited a reasoning strategy associated with the prism. Some students who correctly answered that changing the width of the slit would not change the type of spectrum gave explanations suggesting they were (d) treating the prism as always yielding a continuous spectrum. All of these patterns of reasoning are discussed in detail below.

\section{(a)Confusing a discrete line spectrum and a diffraction pattern}

About half of the students who stated that changing the slit width would give discrete spectrum seemed to be confusing a discrete line spectrum with a single-slit pattern.

If the slit is small enough, we should see some single slit interference in the spectrum, resulting in a discrete spectrum. [UW Honors, task 1]

If the slit were wide enough to get diffraction, then light of certain frequencies would get canceled out in places, leaving spaces between the colors in the spectrum. [UW Intro, task 1]

Often, these students appeared to be thinking that the bright regions of a diffraction pattern correspond to spectral lines. We considered the possibility that some of these students were not familiar with the term "discrete line spectrum" (despite in all cases having had lectures, and often demonstrations and/or labs, on this topic). Later versions of the task included a diagram illustrating a discrete line spectrum (see the version in Fig. 1); there was, however, no discernable impact on the results.

\section{(b)Treating the slit as if it "blocks" certain wavelengths}

On question $1 \mathrm{~A}$, about $10 \%$ of the students who said that changing the width of the slit would give a discrete spectrum explained that narrowing the slit would prevent some wavelengths of light from passing through it. Some based their answers on the idea that certain wavelengths could not "fit" through the slit depending on the relative sizes of the slit and wavelength.
[Changing the slit width could result in a discrete line spectrum] because different color light has different wavelength. With the changing of the slit, some colors can cross the slit, and some cannot. [UW Intro, task 1]

The width of the slit has to be at least as wide as the wavelength of the incident light waves for them to pass through. Since the incident light has waves of varying wavelength, you could change the slit width so that the larger wavelength colors could not pass through while other still could. [UW Intro, task 1]

The tendency to think of a slit as "blocking" some wavelengths of light has been documented previously in the context of physical optics [4].

(c)Tendency to treat a continuous spectrum as consisting of a finite set of colors

About $20 \%$ of the students who said that changing the width of the slit could give a discrete line spectrum reasoned that a narrower slit would make the continuous pattern on the screen wider and thus "separate" the colors from one another. Some seemed to be thinking about how, for diffraction, narrowing a slit widens the diffraction pattern. Although this is correct for diffraction, it does not apply to this experiment. The students went on to predict that since the pattern would become wider, it would either become discrete or the discrete nature of the pattern would become apparent.

"The narrower the slit, the greater the distance between maxima and maxima and we will get line spectrum, ... if [instead] we make the slit wider the spectrum will remain continuous." [UZ Intro, task 1]

"If we decrease the width of the slit, we can potentially spread out the continuous spectrum into segmented or discrete colored fringes because the angle for each colored fringe would increase and result in a more spread out pattern." [UW Intro, task 1]

Often these students seemed to associate each region on the screen that corresponds to a generic color (green, red, blue, etc.) with a single "line" in a discrete line spectrum. They predicted that if the spectrum were widened by changing the slit width, then these (wide) lines would move apart.

The tendency of students to treat different "shades" of a color as if they were the same is reminiscent of an error described in an earlier paper in this investigation [1], in which we found that for a discrete line spectrum, some students treated two different lines with the same "generic" color (e.g., different shades of green) as corresponding to a single electron transition in a gas discharge tube. 
(d)Treating the prism as always yielding a continuous spectrum

About $10 \%$ of the students who answered correctly that changing the slit width would not yield a discrete spectrum based their reasoning on the role of the prism. Most of these students seemed to think that a prism always yields a continuous spectrum.

"The light will still hit the prism as it does now, and the prism will diffract the light into a continuous spectrum always."[UW Intro, task 1]

"No matter what the width space is, there will still be a form of continuous spectrum because of the prism effect." [UW Intro, task 1]

\section{Reasoning elicited by removing the prism (question 1B)}

In question $1 \mathrm{~B}$, students are shown a continuous spectrum and asked whether removing the prism would result in a discrete line spectrum. Only between $20 \%$ and $40 \%$ of the students in the regular sections of the introductory course answered correctly that the spectrum would remain continuous. However, only about one-third of them gave the correct prediction that only a central white region would be visible. Many of the remaining students who gave the correct answer went on to state that a single-slit diffraction pattern would be observed, even though the figure in task 1 shows no evidence of diffraction.

Of the students who answered incorrectly that the pattern would become discrete, about $45 \%$ based their reasoning on single-slit diffraction. A few $(<5 \%)$ gave reasoning consistent with the belief that a prism always yields a continuous spectrum.

Because in question 1B so many students discussed the role of the slit (diffraction) when the prism was removed, we designed task 2 to try to focus their attention on the prism. In task 2, students are asked how removing the prism would affect the pattern on the screen for an experiment that initially shows the discrete pattern formed by a setup involving a gas discharge tube, slit, prism, and screen.

In answering task 2, some students still focused on the slit and many (a) seemed to confuse the discrete line spectrum and a diffraction pattern. Of those who did focus on the prism, some seemed to think (b) that the prism simply serves to "spread out" or "enhance" the pattern, making it easier to observe the pattern that would have appeared on the screen without the prism. Many argued (c) that the prism adds color to light, but that it would not change the overall shape of the pattern observed on the screen. In both of these latter two cases, the students failed to recognize that the fundamental role of the prism in this experiment is to take a narrow beam that would illuminate a single location on the screen and refract the light in slightly different directions according to the wavelength.
(a)Confusing a discrete line spectrum and a diffraction pattern

As stated previously, in both tasks 1 and 2, students often focused on the slit when asked about the effect of removing the prism. On question 1B, about half of the students who thought that removing the prism would result in a discrete spectrum attributed their answer to diffraction caused by the slit. Similar to how students reasoned when the slit width was changed, many seemed to confuse a diffraction pattern with a discrete line spectrum.

"Removing the prism results in a single slit diffraction pattern, where there are places of minima (destructive interference), so the pattern on the screen may be discrete." [UW Intro, task 1]

"It is possible that when we take away the prism, some of the light waves that travel through the slit in the mask will destructively interfere with one another. Though not all light waves will destructively interfere, we should still see some discrete interference lines since some of the light waves will be out of phase with some other light wave from the same source." [UW Intro, task 1]

On task 2, which originally showed a discrete line spectrum, about $20 \%$ of the students stated that a diffraction pattern would be formed on the screen when the prism was removed, even though the original spectrum showed no evidence of diffraction.

"Removing the prism would result in a diffraction pattern ...based on... [the] wavelengths in relation to the width of the single slit." [UW Intro, task 2]

"I think the prism caused diffraction that allows for the colors. Otherwise, there would not be colors, only light, and it would look like a single slit pattern." [UW Intro, task 2]

(b)Treating the prism as serving to enhance a preexisting pattern

For both tasks 1 and 2, some students seemed to think that the prism was serving only to "spread out" the pattern on the screen, making the discrete nature of the pattern more evident.

"The prism serves to bend each color slightly differently. This separates the lines out more so they can be seen better. As a result, if the prism were removed the lines would become more closely spaced." [UW Intro, task 2]

"The prism is bending the light that is going through the single slit. Thus, light with differing frequency is separated respectively to its value. With the removal of the prism, this separation still occurs but it is less obvious with the absence of the prism as the prism was 
just further separating the differing color frequencies." [UW Intro, task 2]

In some cases, the pattern that students thought the prism was serving to spread out was a diffraction pattern from the slit.

"The only thing the prism does is separate the colors in the incoming light. It is the slit that creates the bands." [UW Intro, task 2]

(c)Treating a prism as "adding" color to light without changing the pattern on a screen

On both tasks 1 and 2, some students stated that removing the prism would only change the color of the pattern. Their responses are consistent with thinking that the role of the prism is to "add" color to light. This response was most evident on task 2 , for which the original spectrum was discrete. About $15 \%$ of the students gave explanations consistent with this idea.

"Because the prism just gives colors to the lines, [the] lines will be in the same spot." [UW Intro, task 2]

"A prism breaks monochromatic light into different colors so if the prism is removed all the light will be one color." [UW Intro, task 2]

"The lines would still be in the same relative location due to the single slit, but the colors would disappear due to the fact that the prism is no longer there to diffract the light and create the colors." [UW Intro, task 2]

These students typically did not describe any changes to the overall shape of the pattern when the prism is removed but treated the shape as independent of the presence of the prism.

\section{Reasoning elicited by changing the prism-mask distance (question 1C)}

On question $1 \mathrm{C}$, students were asked whether the continuous spectrum shown could become a discrete line spectrum if the distance between the prism and screen were changed. Only about $40 \%$ answered correctly. Most of them used one of two reasoning strategies: recognizing that the type of spectrum depends only on the light source or correctly describing how the continuous spectrum would become more "spread out" or "closer together" depending on whether the prism-mask distance increases or decreases, respectively.

About $20 \%-30 \%$ of the students in the regular sections of the introductory sequence incorrectly stated that changing the prism-screen distance could change the original continuous spectrum into a discrete line spectrum. About half of them seemed to treat the given continuous spectrum (coming from an incandescent bulb) as if it had a finite number of colors or wavelengths. Others gave explanations in which they seemed to be confusing the discrete line spectrum with a diffraction pattern or reasoned that the prism always yields a continuous spectrum. These latter two types of explanations are not included here as they have been discussed previously.

(a)Tendency to treat a continuous spectrum as consisting of a finite set of colors

Often, the students who predicted that changing the prism-screen distance could result in a discrete spectrum $(45 \%)$ seemed to be treating the continuous pattern from the incandescent bulb as having gaps that were too small to be seen in the original pattern. They predicted that these gaps would become more apparent by increasing the prismscreen distance.

"If the distance is great enough between the prism and the screen, the separation between each spectrum line will be great enough to distinguish each individually." [UW Intro, Q1]

"The screen might be too close. Bringing the screen back could allow for discrete colors to be seen." [UW Intro, Q1]

The tendency to treat the continuous spectrum as being discrete also arose when students were asked to predict the effect of changing the slit size (question 1A) or when the prism was removed (question 1B).

\section{Reasoning elicited by replacing the prism by a diffraction grating (question 1D)}

In question 1D, students were asked whether the continuous spectrum would become a discrete spectrum if the prism were replaced by a diffraction grating. We asked this question since a grating is commonly used in experiments that illustrate spectral lines. Only about $20 \%$ of the students answered correctly with correct reasoning: either stating that the type of pattern depends only on the source $(15 \%)$ or reasoning about the resulting (complicated) diffraction pattern for incoming light that consists of a wide range of wavelengths (about 5\%).

Most of the students answered incorrectly. The most common reasoning patterns include (a) confusing a line spectrum with diffraction due to an optical grating, (b) treating a grating as if it "blocks" or "filters out" certain wavelengths and (c) treating a continuous spectrum as consisting of a finite set of colors.

Some of the incorrect reasoning patterns already discussed also arose (e.g., treating the prism as always yielding continuous spectrum). On this question in particular, students often gave answers that suggested a memorized or misremembered response. For example, some simply stated that an optical grating would not yield a spectrum (5\%). In some cases, they seemed to be 
treating white light as consisting of a single wavelength. Others claimed that they had observed in lab that a diffraction grating always yields a discrete spectrum $(5 \%)$.

(a)Confusing line spectra with diffraction patterns due to optical gratings

Between $40 \%$ and $70 \%$ of the UW introductory students incorrectly stated that the continuous spectrum would become discrete if the prism was replaced by an optical grating. About a quarter of them did not seem to be distinguishing between a discrete spectrum and a diffraction pattern.

[A discrete spectrum would appear if the prism were replaced by an optical grating. An]"optical grating has mins/maxes instead of a continuous spread." [UW Intro, task 1]

"An optical grating would allow for certain discrete spectrum because the light would undergo points of constructive and destructive interference." [UW Honors, task 1]

"The grating will create interference pattern on the screen, which will be a discrete spectrum." [UW Intro, task 1]

"This would cause some of the wavelengths to cancel out (be minima) and some to be bright (maxima) which would give the discrete spectra." [UW Intro, task 1]

These students seemed to recall that a diffraction grating yields a pattern of bright and dark regions, but incorrectly associated this pattern with a discrete line spectrum. Interpretation of some student responses was made difficult by the fact that, despite having seen both patterns in class, some students did not seem to know what was meant by the terms "discrete spectrum" or "line spectrum." As stated previously, on some versions of the task we included a diagram illustrating what was meant by the term discrete line spectrum. There was, however, no discernable impact on student responses.

\section{(b)Treating the grating as if it blocks of filters out certain} wavelengths

About $10 \%$ of the students who stated that replacing the prism with an optical grating would result in a discrete spectrum, argued that the grating would "filter" or "block" some colors. Similar reasoning had been observed when students were answering about the effect of changing the slit width.

"Using a grating you can allow certain wavelengths to pass through while not allowing others." [UW Intro, task 1]
"Yes [the pattern will become discrete], because a diffraction grating will block off certain wavelengths so only certain sized waves will go through the gratings and will appear on the screen." [UW Intro, task 1]

(c)Tendency to treat a continuous spectrum as consisting of a finite set of colors

On question 1D, about replacing the prism with a diffraction grating, about $15 \%$ of the students gave answers that suggested that they were thinking of the continuous spectrum as if it consisted of a finite set of colors. The responses were similar to those we saw when they were asked about the changes to the slit width and changes to the prism-screen distance.

"This could again increase the angle of diffraction to a point where the lines are distinguishable." [UW Intro, task 1]

"The diffraction grating would bend the various wavelengths more, resulting in a discrete spectrum" [UW Intro, task 1]

\section{Reasoning elicited by replacing the light source (question $1 E$ )}

The final part of task 1 asked students whether changing the light source in the original experiment (Fig. 1) could result in a discrete line spectrum. In fact, this change is the only one that could do so. Thus, this part of the task is perhaps the most direct probe of student understanding of the formation of discrete line spectra. Although the majority of students answered correctly (50\%-75\%), only between $5 \%$ and $30 \%$ of the students identified this change as the only one that could yield a discrete spectrum. Although in many cases, students identified a specific light source, only about $65 \%$ of them gave one that would, in fact, yield a discrete spectrum.

In some cases, the errors made by students were directly associated with their ideas about light and light sources. In other cases, the question elicited ideas about the role of other optical elements in the experiment (the slit, the prism, or the overall experimental setup). In many cases, the latter strategies were similar to those discussed in earlier sections of this article, but revealed additional insights into their nature of prevalence.

\section{Reasoning associated with the role of the light source}

To answer question $1 \mathrm{E}$ about whether changing the light source could result in a discrete spectrum, students needed to recall what they had previously been taught about atomic spectra and the sources that emit light of discrete wavelengths or energies. However, many students $(\sim 35 \%)$ stated that the pattern on the screen is independent of the light source. Others $(\sim 10 \%)$ seemed to think that a discrete 
pattern requires a light source that emits only a single wavelength.

\section{(a)Treating all light sources as they are same}

Students who said that the pattern is independent of the light source often stated that all light sources have similar spectra. Some stated explicitly that the spectrum is independent of the light source. Others seemed to be treating all sources as having a continuous spectrum, but it was not clear whether they thought the spectra were identical.

"Light is light is light; it [the spectrum] is independent of the source." [UW Intro, task 1]

"[No] All the light sources result in a continuous spectrum." [UW Intro, task 1]

"[No] The new light bulb would just be emitting light like this one." [UW Intro, task 1]

"No, because the other type of light source could have the same wavelength as the light bulb, therefore, it would not result in a discrete spectrum." [UW Intro, task 1]

"[No] The light will still be the same, no matter the source." [UW Intro, task 1]

\section{(b)Treating discrete spectra as monochromatic}

Of the $65 \%$ of the students who correctly stated that a discrete line spectrum could be formed if the incandescent bulb were replaced by another light source, about $15 \%$ went on to say that the source must be monochromatic.

"Replacing the light bulb with a monochromatic light source would result in a discrete spectrum because only one wavelength of light would be present." [UW Intro, Task 1]

A monochromatic source, like a laser, would, in fact, produce a discrete pattern (only one line would appear on the screen). However, students who answered in this way failed to recognize that they had studied another source that produces a discrete pattern. Some of the students even explicitly stated that only monochromatic sources would work. The percentage of students who said the source must be monochromatic was essentially independent of whether or not the task included the picture of a discrete line spectrum (with multiple lines shown) that was provided to remind students of what was meant by the discrete line spectrum.

\section{Reasoning associated with the role of the slit}

Of the students who stated that the light source does not affect the pattern on the screen, some seemed to be confusing a discrete line spectrum with a diffraction pattern.
(c)Confusing a discrete line spectrum and a diffraction pattern

"[No], Changing the light source cannot cause interference patterns." [UW Intro, task 1]

"No, the light bulb does not change the spectrum. Spectrum is defined by these equations. $\mathrm{d} \sin \theta=\mathrm{m} \lambda=$ $\operatorname{asin} \theta$.., i.e., if $\theta$ is really small $=y / \mathrm{L}$." [UW Intro, task 1]

Other students reasoned that the original source does not matter because it is the slit that determines the pattern on the screen. In most cases, these students did not seem to be regarding the slit as simply columnating the beam, but instead treated it as being narrow enough for a diffraction pattern with maxima and minima to be visible on the screen.

[No] ... the spectrum is considered to come from the slit (as the source of light) rather than from the bulb itself. [UW Intro, task 1]

[No] The light coming from the slit would still be made from infinitely small point sources and so even with a different type of source, there would still be a continuous spectrum. [UW Intro, task 1]

\section{Reasoning associated with the role of the prism}

(d)Treating a prism as always yielding a continuous spectrum

Many of the students who said that the light source does not affect the pattern on the screen also explained that light passing through a prism always yields a continuous spectrum-independent of the light source. Some seemed to treat the prism as being solely responsible for producing the spectrum.

"[Changing the source will not change the spectrum, because] the spectrum is produced by the prism, not the light source." [UW Intro, task 1]

Other students recognized that the light source plays a role in the colors or wavelengths present in light but, regardless, seemed to think that a prism would produce a continuous spectrum.

"As long as the prism is there, I think it doesn't matter if you change the light source. It'll still give us continuous spectrum, maybe of different widths." [UW Honors, task 1]

"The light would still contain a spectrum of wavelengths that would be made continuous by the prism, regardless of what the spectrum itself actually was." [UW Intro, task 1] 
"[No] The prism would still diffract any wavelengths sent to it, so any different source would still be subject to continuous diffraction of whatever it's wavelength is." [UW Intro, task 1]

\section{Reasoning associated with other unspecified elements in the experiment}

Not all students who stated that the pattern on the screen is independent of the light source indicated which of the elements is responsible for creating the pattern. Often, they referred to the experimental setup in general.

"This [changing the light source] will not change anything because it will just make a different wavelength of light and have no effect on the type of spectrum." [UW Intro, Task 1]

"The bulb isn't what causes the spectrum it [is] the difference in object in front of the light source [between the source and screen] that splits it into the spectrum." [UW Intro, Task 1]

"A different type of source would produce the same type of pattern. You would need a special type of viewing device, such as a spectrometer, to see a discrete spectrum." [UW Intro, Task 1]

General failure to recognize that the source determines whether or not a spectrum is discrete

About $25 \%$ of all the students who stated that changing the light source could not give a discrete spectrum explicitly stated that the type of the source does not play a role. Often, they were quite explicit.

"The discrete spectrum can happen only if there is something to bend the light and separate them depending on their wavelengths. [The] light source doesn't matter." [UW Intro; task 1]

"The type of source does not matter, only the slit/ grating/prism." [UW Intro; task 1]

A general failure to understand the unique role of the light source in yielding a discrete spectrum, was implicit, if not explicit, in the majority of responses given by students to all questions. As shown in Table III, only $30 \%$ or fewer of the students in all the introductory courses indicated on task 1 that only choice $\mathrm{E}$ (changing the light source) could result in a discrete line spectrum.

\section{CONCLUSION}

The results presented in this paper are part of a broad investigation into student understanding of the atomic model used to relate discrete line spectra and electron transitions. Earlier papers examined the ability of students to associate specific transitions with specific lines in a discrete spectrum [1,2]. This paper describes an assessment of student understanding of spectra through the use of an experimental setup (a light source, slit, prism or grating, and screen) that is often used to demonstrate discrete line spectra.

The tasks used in this study ask students to predict how basic changes to the experimental setup (e.g., removing the prism, changing the size of the slit, or changing the light source) will affect the pattern that appears on the screen. A picture emerged suggesting that after instruction, as many as $80 \%$ of the students in a typical introductory physics course do not associate a discrete spectrum with the light source. There was widespread confusion about the formation and structure of such spectra.

In many cases, student responses suggest a failure to distinguish between various optical phenomena and between the patterns (discrete, continuous, and diffraction) they produce on a screen. For example, many students seemed to regard discrete spectra as a type of diffraction pattern, often conflating the minima and maxima for diffraction with the dark regions and bright lines in a discrete spectrum. Others seemed to treat the generic colors in a continuous spectrum as if they were discrete (broad) lines, but with the spacing so close that it is not possible to see the dark regions between them. Still others gave answers in which they treated continuous and discrete emission spectra as if they can be transformed from one into the other simply by changing the optical devices that are used to observe them. Even juniors and graduate TAs gave answers that are consistent with these ways of thinking.

Other student responses suggest specific difficulties associated with the function of individual optical elements. For example, students often treated a prism as adding color to light or stated that a prism always results in a continuous spectrum. Other students treated a slit or grating as blocking or filtering certain wavelengths of light.

Experienced instructors might expect that students would confuse discrete line spectra with other optical patterns, especially since in a typical course, students have been quickly introduced to a wide variety of optical phenomena. The patterns these produce can appear superficially similar. However, the extent of the confusion, and the ways in which students relate the different patterns, may be surprising. Moreover, taken together, all of these different reasoning patterns can be regarded as indicating that after explicit instruction, many students have failed to understand a key idea underlying the model for electron transitions in atoms: that discrete emission spectra are associated with light composed of only a finite number of wavelengths.

Students are unlikely to possess firm alternative ideas regarding formation of spectra, since the phenomena are not part of their common experience. It therefore seems likely that, when asked to construct answers and 
explanations to questions about discrete spectra, they use both existing and recently acquired knowledge. This process can also be influenced by visual and verbal cues in the tasks. For example, the presence of a slit in the experiment may have led some students to treat these tasks as diffraction problems. Similarly, students might associate a prism with a continuous spectrum, prompting them to conclude that experiments involving prisms cannot produce discrete spectra. This interpretation of the results is consistent with a theory of activation of cognitive resources and may reflect the extent to which students had not yet formed coherent mental models regarding discrete, continuous, or diffraction spectra [23]. As can be seen by the student responses in this paper, the explanations often reveal a high level of confusion about the basic concepts and phenomena of wave optics and line spectra. Moreover, many students were not consistent in their answers when asked about the effects resulting from different changes to the various devices.

The issues we have identified are also consistent with some of the possible reasons that Roth et al. [18] have described for why students often do not learn from traditional lecture demonstrations. They may lack a theoretical framework that can help them separate the phenomenon (in this case discrete line spectra) from noise (e.g., other patterns they have observed and the functions of the various parts of the experimental setup). Students' answers also suggest "interference" related to the discourse used in wave optics and modern physics, as well from their recall of other demonstrations and images that have surface resemblance.
Students who have not developed a functional understanding of wave optics and discrete line spectra may regard the different patterns they have observed as being essentially similar and even interpret a continuous spectrum as consisting of a series of broad, colored "lines." It is therefore not surprising that so many difficulties are evident in their explanations.

The results of this study can help remind physics instructors that typical demonstrations or experiments may be significantly misunderstood by students, even leading them to form incorrect mental models of the phenomena being demonstrated. Students need explicit help in recognizing the key aspects of the different phenomena in question, in differentiating between the different patterns they observe on a screen, and in becoming aware of the roles that the different elements play in the experimental setup. The patterns in student thinking and reasoning revealed by this research can serve to guide the development of curriculum that helps students make sense of what they observe. However, such approaches necessarily require more time than is usually allocated to the topics of wave optics and line spectra in a typical course.

\section{ACKNOWLEDGMENTS}

This work was supported in part by National Science Foundation Grant No. 1022449 and 1821032 . The authors gratefully thank the faculty who welcomed this research into their classrooms. Open access funding provided by the University of Vienna.
[1] L. Ivanjek, P. S. Shaffer, L. C. McDermott, M. Planinic, and D. Veza, Research as a guide for curriculum development: An example from introductory spectroscopy. I. Identifying student difficulties with atomic emission spectra, Am. J. Phys. 83, 85 (2015).

[2] L. Ivanjek, P. S. Shaffer, L. C. McDermott, M. Planinic, and D. Veza, Research as a guide for curriculum development: An example from introductory spectroscopy. II. Addressing student difficulties with atomic emission spectra, Am. J. Phys. 83, 171 (2015).

[3] S. Lee, Students' understanding of spectra, Ph.D. thesis, Kansas State University, 2002.

[4] B. S. Ambrose, P. S. Shaffer, R. N. Steinberg, and L. C. McDermott, An investigation of student understanding of single-slit diffraction and double-slit interference, Am. J. Phys. 67, 146 (1999).

[5] B. S. Ambrose, P. R. L. Heron, S. Vokos, and L. C. McDermott, Student understanding of light as an electromagnetic wave: Relating the formalism to physical phenomena, Am. J. Phys. 67, 891 (1999).
[6] S. Vokos, P. S. Shaffer, B.S. Ambrose, and L. C. McDermott, Student understanding of the wave nature of matter: Diffraction and interference of particles, Am. J. Phys. 68, S42 (2000).

[7] K. Wosilait, P. R. L. Heron, P. S. Shaffer, and L. C. McDermott, Addressing student difficulties in applying a wave model to the interference and diffraction of light, Am. J. Phys. 67, S5 (1999).

[8] R. B. Ward, P. M. Sadler, and I. I. Shapiro, Learning physical science through astronomy activities: A comparison between constructivist and traditional approaches in grades 3-6, Astron. Educ. Rev. 6, 1 (2007).

[9] C. W. Anderson and E. L. Smith, Children's conceptions of light and color: Understanding the role of unseen rays (Report No. 400-81-0014). East Lansing, MI: Institute for Research on Teaching. (ERIC Reproduction Document No. ED 270318), https://eric.ed.gov/?id=ED270318.

[10] C. Wieman and N. G. Holmes, Measuring the impact of an instructional laboratory on the learning of introductory physics, Am. J. Phys. 83, 972 (2015). 
[11] N. G. Holmes, J. Olsen, J. L. Thomas, and C. E. Wieman, Value added or misattributed? A multi-institution study on the educational benefit of labs for reinforcing physics content, Phys. Rev. Phys. Educ. Res. 13, 010129 (2017).

[12] I. Abrahams and R. Millar, Does practical work really work? A study of the effectiveness of practical work as a teaching and learning method in school science, Int. J. Sci. Educ. 30, 1945 (2008).

[13] B. M. Zwickl, N. Finkelstein, and H. J. Lewandowski, The process of transforming an advanced lab course: Goals, curriculum, and assessments, Am. J. Phys. 81, 63 (2013).

[14] C. Crouch, A. P. Fagen, J. P. Callan, and E. Mazur, Classroom demonstrations: Learning tools or entertainment?, Am. J. Phys. 72, 835 (2004).

[15] P. A. Kraus, Promoting active learning in lecture-based courses: demonstrations, tutorials, and interactive tutorial lectures, Ph.D. thesis, University of Washington, 1997.

[16] K. Miller, N. Lasry, K. Chu, and E. Mazur, Role of physics lecture demonstrations in conceptual learning, Phys. Rev. ST Phys. Educ. Res. 9, 020113 (2013).

[17] D. R. Sokoloff and R. K. Thornton, Using interactive lecture demonstrations to create an active learning environment, Phys. Teach. 35, 340 (1997).

[18] W.-M. Roth, C. J. McRobbie, K. B. Lucas, and S. Boutonn, Why may students fail to learn from demonstrations? A social practice perspective on learning in physics, J. Res. Sci. Teach. 34, 509 (1997).
[19] A. Northedge, Organizing excursions into specialist discourse communities: A sociocultural account of university teaching, in Learning for Life in the 21st Century, edited by G. Wells and G. Claxton (Blackwell Publishing Ltd, Oxford, UK, 2002), pp. 252-264.

[20] T. Fredlund, C. Linder, J. Airey, and A. Linder, Unpacking physics representations: Towards an appreciation of disciplinary affordance, Phys. Rev. ST Phys. Educ. Res. 10, 020129 (2014).

[21] A. A. diSessa, Toward an epistemology of physics, Cognit. Instr. 10, 105 (1993).

[22] M. C. Linn, B. Eylon, and E. A. Davis, The knowledge integration perspective on learning, in Internet Environments for Science Education, edited by M.C. Linn, E. A. Davis, and P. Bell (Lawrence Erlbaum Associates, Mahwah, NJ, 2004).

[23] D. Hammer, A. Elby, R. E. Scherr, and E. F. Redish, Resources, framing, and transfer, in Transfer of Learning from a Modern Multidisciplinary Perspective, edited by J. Mestre (Information Age Publishing, Greenwich, CT, 2005) 89-120.

[24] L. C. McDermott and P. S. Shaffer, Tutorials in Introductory Physics, 1st ed. (Prentice Hall, Upper Saddle River, NJ, 2002).

[25] M. T. H. Chi, Quantifying qualitative analyses of verbal data: A practical guide, J. Learn. Sci. 6, 271 (1997). 